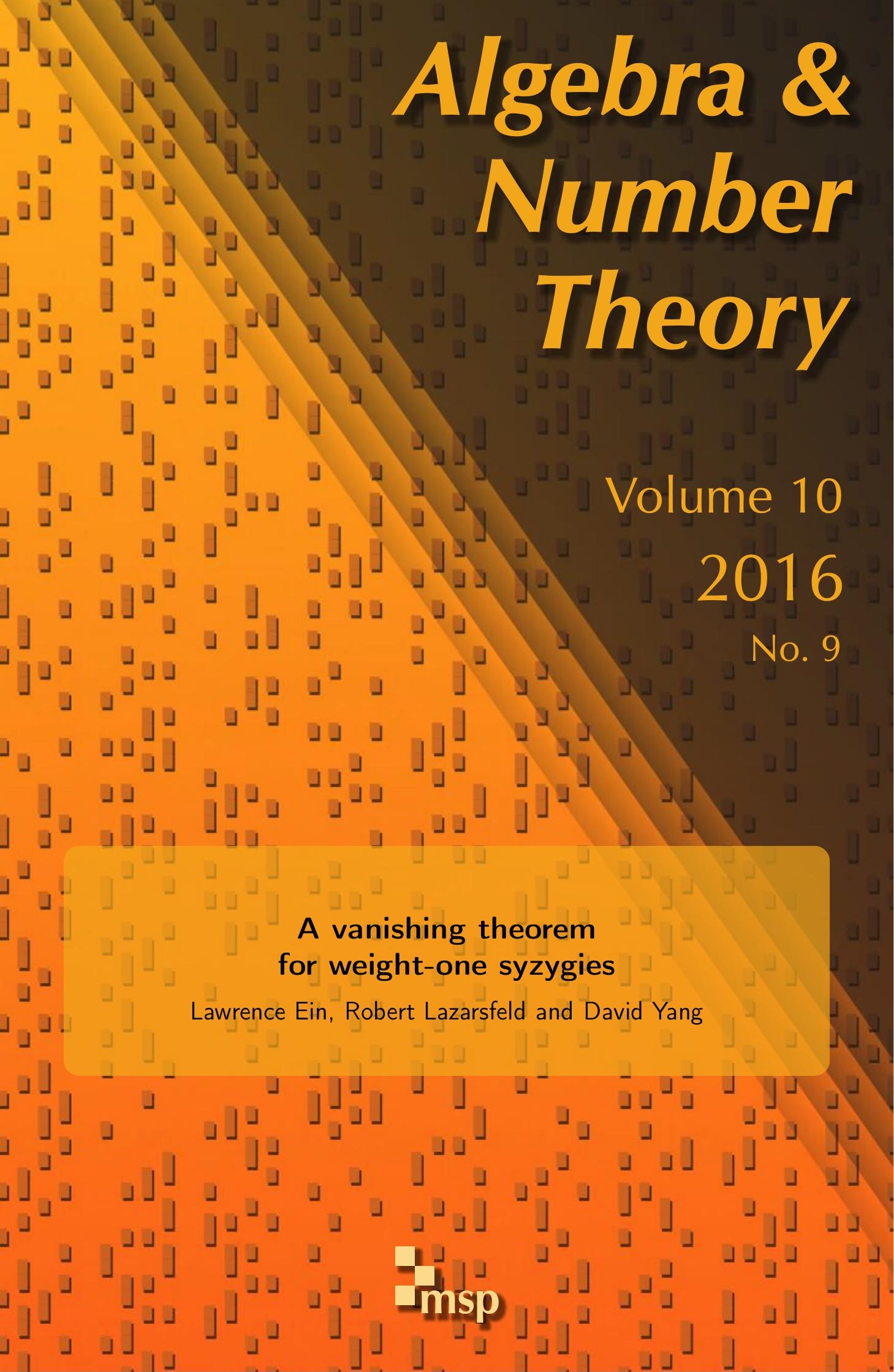




\title{
A vanishing theorem for weight-one syzygies
}

\section{Lawrence Ein, Robert Lazarsfeld and David Yang}

\begin{abstract}
We give a criterion for the vanishing of the weight-one syzygies associated to a line bundle $B$ in a sufficiently positive embedding of a smooth complex projective variety of arbitrary dimension.
\end{abstract}

\section{Introduction}

Inspired by the methods of Voisin [2002; 2005], Ein and Lazarsfeld [2015] recently proved the gonality conjecture of [Green and Lazarsfeld 1986], asserting that one can read off the gonality of an algebraic curve $C$ from the syzygies of its ideal in any one embedding of sufficiently large degree. They deduced this as a special case of a vanishing theorem for the asymptotic syzygies associated to an arbitrary line bundle $B$ on $C$, and conjectured that an analogous statement should hold on a smooth projective variety of any dimension. The purpose of this note is to prove the conjecture in question.

Turning to details, let $X$ be a smooth complex projective variety of dimension $n$, and set

$$
L_{d}=d A+P,
$$

where $A$ is ample and $P$ is arbitrary. We always assume that $d$ is sufficiently large so that $L_{d}$ is very ample, defining an embedding

$$
X \subseteq \mathbb{P} H^{0}\left(X, L_{d}\right)=\mathbb{P}^{r_{d}} .
$$

Given an arbitrary line bundle $B$ on $X$, we wish to study the weight-one syzygies of $B$ with respect to $L_{d}$ for $d \gg 0$. More precisely, let $S=\operatorname{Sym} H^{0}\left(X, L_{d}\right)$ be the homogeneous coordinate ring of $\mathbb{P} H^{0}\left(X, L_{d}\right)$, and put

$$
R=R\left(X, B ; L_{d}\right)=\bigoplus_{m} H^{0}\left(X, B+m L_{d}\right)
$$

The research of Ein was partially supported by NSF grant DMS-1501085. Lazarsfeld's research was partially supported by NSF grant DMS- 1439285 .

MSC2010: primary 14J99; secondary 13D02.

Keywords: Syzygies. 
Thus $R$ is a finitely generated graded $S$-module, and hence has a minimal graded free resolution $E_{\bullet}=E_{\bullet}\left(B ; L_{d}\right)$ :

$$
0 \longrightarrow E_{r_{d}} \longrightarrow \cdots \longrightarrow E_{2} \longrightarrow E_{1} \longrightarrow E_{0} \longrightarrow R \longrightarrow 0
$$

where $E_{p}=\bigoplus S\left(-a_{p, j}\right)$. As customary, denote by $K_{p, q}\left(X, B ; L_{d}\right)$ the finitedimensional vector space of degree $p+q$ minimal generators of $E_{p}$, so that

$$
E_{p}\left(B ; L_{d}\right)=\bigoplus_{q} K_{p, q}\left(X, B ; L_{d}\right) \otimes_{\mathbb{C}} S(-p-q) .
$$

We refer to elements of this group as $p$-th syzygies of $B$ with respect to $L_{d}$ of weight $q$. When $B=\mathcal{O}_{X}$ we write simply $K_{p, q}\left(X ; L_{d}\right)$, which — provided that $d$ is large enough so that $L_{d}$ is normally generated - are the vector spaces describing the syzygies of the homogeneous ideal $I_{X} \subset S$ of $X$ in $\mathbb{P} H^{0}\left(X, L_{d}\right)$.

The question we address involves fixing $B$ and asking when it happens that

$$
K_{p, q}\left(X, B ; L_{d}\right)=0 \text { for } d \gg 0 .
$$

When $q=0$ or $q \geq 2$ the situation is largely understood thanks to results of Green [1984a; 1984b] and Ein and Lazarsfeld [1993; 2012]. (See Remark 1.10 for a summary.) Moreover in this range the statements are uniform in nature, in that they don't depend on the geometry of $X$ or $B$. However as suggested in [Ein and Lazarsfeld 2012, Problem 7.1], for $K_{p, 1}\left(X, B ; L_{d}\right)$ one can anticipate more precise asymptotic results that do involve geometry. This is what we establish here.

Recall that a line bundle $B$ on a smooth projective variety $X$ is said to be $p$-jet very ample if for every effective zero-cycle

$$
w=a_{1} x_{1}+\cdots+a_{s} x_{s}
$$

of degree $p+1=\sum a_{i}$ on $X$, the natural map

$$
H^{0}(X, B) \rightarrow H^{0}\left(X, B \otimes \mathcal{O}_{X} / \mathfrak{m}_{1}^{a_{1}} \cdots \mathfrak{m}_{s}^{a_{s}}\right)
$$

is surjective, where $\mathfrak{m}_{i} \subseteq \mathcal{O}_{X}$ is the ideal sheaf of $x_{i}$. So for example if $p=1$ this is simply asking that $B$ be very ample. When $\operatorname{dim} X=1$ the condition is the same as requiring that $B$ be $p$-very ample - i.e., that every subscheme $\xi \subset X$ of length $p+1$ imposes independent conditions in $H^{0}(X, B)$ - but in higher dimensions it is a stronger condition.

Our main result is

Theorem A. If B is p-jet very ample, then

$$
K_{p, 1}\left(X, B ; L_{d}\right)=0 \quad \text { for } d \gg 0 .
$$


The statement was conjectured in [Ein and Lazarsfeld 2015, Conjecture 2.4], where the case $\operatorname{dim} X=1$ was established.

It is not clear whether one should expect that $p$-jet amplitude is equivalent to the vanishing of $K_{p, 1}\left(X, B ; L_{d}\right)$ for $d \gg 0$. However we prove:

Theorem B. Suppose that there is a reduced $(p+1)$-cycle $w$ on $X$ that fails to impose independent conditions on $H^{0}(X, B)$. Then

$$
K_{p, 1}\left(X, B ; L_{d}\right) \neq 0 \quad \text { for } d \gg 0 .
$$

In general, the proof of Theorem A will show that if $H^{1}(X, B)=0$, then the jet amplitude hypothesis on $B$ is equivalent when $d \gg 0$ to the vanishing of a group that contains $K_{p, 1}\left(X, B ; L_{d}\right)$ as a subspace (Remark 1.8).

When $B=K_{X}$ is the canonical bundle of $X$, Theorem A translates under a mild additional hypothesis into a statement involving the syzygies of $L_{d}$ itself.

Corollary C. Assume that $H^{i}\left(X, \mathcal{O}_{X}\right)=0$ for $0<i<n$, or equivalently that $X \subseteq \mathbb{P}^{r_{d}}$ is projectively Cohen-Macaulay for $d \gg 0$.

(i) The canonical bundle $K_{X}$ of $X$ is very ample if and only if

$$
K_{r_{d}-n-1, n}\left(X ; L_{d}\right)=0 \text { for } d \gg 0 .
$$

(ii) If $K_{X}$ is $p$-jet very ample, then

$$
K_{r_{d}-n-p, n}\left(X ; L_{d}\right)=0 \text { for } d \gg 0 .
$$

When $n=\operatorname{dim} X=1$, this (together with Theorem B) implies $K_{r_{d}-c, 1}\left(X ; L_{d}\right) \neq 0$ for $d \gg 0$ if and only if $X$ admits a branched covering $X \rightarrow \mathbb{P}^{1}$ of degree $\leq c$, which is the statement of the gonality conjecture established in [Ein and Lazarsfeld 2015].

The proof of Theorem A occupies Section 1. It follows very closely the strategy of [Ein and Lazarsfeld 2015], which in turn was inspired by the ideas of Voisin [2002; 2005]. However instead of working on a Hilbert scheme or symmetric product, we work on a Cartesian product of $X$, using an idea that goes back in a general way to Green [1984b]. For the benefit of nonexperts, we outline now the approach in some detail in the toy case $p=0 .{ }^{1}$

Keeping notation as above, it follows from the definition that $K_{0,1}\left(X, B ; L_{d}\right)=0$ if and only if the multiplication map

$$
H^{0}(X, B) \otimes H^{0}\left(X, L_{d}\right) \rightarrow H^{0}\left(X, B \otimes L_{d}\right)
$$

${ }^{1}$ This was in fact the train of thought that led us to the arguments here and in [Ein and Lazarsfeld 2015]. 
is surjective: in fact, $K_{0,1}$ is its cokernel. A classical way to study such maps is to pass to the product $X \times X$ and then restrict to the diagonal. Specifically, $(*)$ is identified with the homomorphism

$$
H^{0}\left(X \times X, \operatorname{pr}_{1}^{*} B \otimes \operatorname{pr}_{2}^{*} L_{d}\right) \rightarrow H^{0}\left(X \times X, \operatorname{pr}_{1}^{*} B \otimes \operatorname{pr}_{2}^{*} L_{d} \otimes \mathcal{O}_{\Delta}\right)
$$

arising from this restriction. Thus the vanishing $K_{0,1}\left(X, B ; L_{d}\right)=0$ is implied by the surjectivity of $(* *)$. Green [1984b] observed that there is a similar way to tackle the $K_{p, 1}$ for $p \geq 1$ : one works on the $(p+2)$-fold product $X^{p+2}=X \times X^{p+1}$ and restricts to a suitable union of pairwise diagonals (Proposition 1.1). This is explained in Section 1, and forms the starting point of our argument. Although not strictly necessary we give a new proof of Green's result here that clarifies its relation to other approaches.

There remains the issue of actually proving the surjectivity of $(* *)$ for $d \gg 0$ provided that $B$ is 0 -jet very ample, i.e., globally generated. For this one starts with the restriction

$$
\operatorname{pr}_{1}^{*} B \rightarrow \operatorname{pr}_{1}^{*} B \otimes \mathcal{O}_{\Delta}
$$

of sheaves on $X \times X$ and pushes down to $X$ via $\mathrm{pr}_{2}$. There results a map of vector bundles

$$
\mathrm{ev}_{B}: H^{0}(X, B) \otimes_{\mathbb{C}} \mathcal{O}_{X} \rightarrow B
$$

on $X$ which is given by evaluation of sections of $B$. Note that $\mathrm{ev}_{B}$ is surjective as a map of bundles if and only if $B$ is globally generated. The surjectivity in (*) or $(* *)$ is then equivalent to the surjectivity on global sections of the map

$$
H^{0}(X, B) \otimes L_{d} \rightarrow B \otimes L_{d}
$$

obtained from twisting $\mathrm{ev}_{B}$ by $L_{d}$.

Suppose now that $B$ is 0 -jet very ample. The setting $M_{B}=\operatorname{ker}\left(\mathrm{ev}_{B}\right)$, we get an exact sequence

$$
0 \rightarrow M_{B} \rightarrow H^{0}(X, B) \otimes_{\mathbb{C}} \mathcal{O}_{X} \rightarrow B \rightarrow 0
$$

of sheaves on $X$. Serre vanishing implies that

$$
H^{1}\left(X, M_{B} \otimes L_{d}\right)=0
$$

for $d \gg 0$, and by what we have just said this means that $K_{0,1}\left(X, B ; L_{d}\right)=0$. The proof of Theorem $A$ in general proceeds along analogous lines. We construct a torsion-free sheaf $\mathcal{E}_{B}=\mathcal{E}_{p+1, B}$ of rank $p+1$ on $X^{p+1}$ whose fiber at $\left(x_{1}, \ldots, x_{p+1}\right)$ is identified with

$$
H^{0}\left(X, B \otimes \mathcal{O}_{X} / \mathfrak{m}_{1} \cdots \mathfrak{m}_{p+1}\right)
$$


where $\mathfrak{m}_{i} \subseteq \mathcal{O}_{X}$ is the ideal sheaf of $x_{i}$. This comes with an evaluation map

$$
\operatorname{ev}_{p+1, B}: H^{0}(X, B) \otimes_{\mathbb{C}} \mathcal{O}_{X^{p+1}} \rightarrow \mathcal{E}_{B}
$$

which is surjective (as a map of sheaves) if and only if $B$ is $p$-jet very ample. ${ }^{2}$ Green's criterion for the vanishing of $K_{p, 1}\left(X, B ; L_{d}\right)$ turns out to be equivalent to the surjectivity of the map on global sections resulting from twisting $\operatorname{ev}_{p+1, B}$ by a suitable ample divisor $\mathcal{N}_{d}$ on $X^{p+1}$ deduced from $L_{d}$, and this again follows from Serre vanishing.

Returning to the case $p=0$, the argument just sketched actually proves more. Namely for arbitrary $B$ one has an exact sequence

$$
0 \rightarrow \operatorname{ker}\left(\mathrm{ev}_{B}\right) \rightarrow H^{0}(X, B) \otimes_{\mathbb{C}} \mathcal{O}_{X} \rightarrow B \rightarrow \operatorname{coker}\left(\mathrm{ev}_{B}\right) \rightarrow 0,
$$

and so Serre vanishing shows conversely that if $B$ is not 0 -jet very ample then

$$
K_{p, 1}\left(X, B ; L_{d}\right)=H^{0}\left(X, \operatorname{coker}\left(\mathrm{ev}_{B}\right) \otimes L_{d}\right) \neq 0
$$

for $d \gg 0$. Unfortunately this does not generalize when $p \geq 1$ because the computations on $X^{p+1}$ lead to groups that contain $K_{p, 1}\left(X, B ; L_{d}\right)$ as summands, but may contain other terms as well. (Said differently, Green's criterion is sufficient but not necessary for the vanishing of $K_{p, 1}$.) To prove a nonvanishing statement such as Theorem B, one needs a geometric interpretation of $K_{p, 1}$ itself. Voisin [2002; 2005] achieves this by working on a Hilbert scheme - which has the advantage of being smooth when $\operatorname{dim} X=2$ - while Yang [2014] passes in effect to the symmetric product. ${ }^{3}$ We follow the latter approach for Theorem B: we exhibit a sheaf on $\operatorname{Sym}^{p+1}(X)$ whose twisted global sections compute $K_{p, 1}\left(X, B ; L_{d}\right)$, and we show that it is nonzero provided that there is a reduced cycle that fails to impose independent conditions on $H^{0}(X, B)$. Then we can argue much as in the case $p=0$ just described. This is the content of Section 2.

\section{Proof of Theorem A}

This section is devoted to the proof of Theorem A from the introduction.

We start by describing the set-up. As above, $X$ is a smooth complex projective variety of dimension $n$, and we consider the $(p+2)$-fold product

$$
Y \stackrel{\text { def }}{=} X \times X^{p+1}
$$

of $X$ with itself. For $0 \leq i<j \leq p+1$ denote by

$$
\pi_{i, j}: Y \rightarrow X \times X
$$

${ }^{2}$ Note that the fiber of $B$ at a point $x \in X$ is identified with $B \otimes \mathcal{O}_{X} / \mathfrak{m}_{x}$, i.e., $\mathcal{E}_{1, B}=B$.

${ }^{3}$ Roughly speaking, one is picking out $K_{p, 1}$ inside Green's construction as the space of invariants under a suitable action of the symmetric group. 
the projection of $Y$ onto the product of the $i$ and $j$ factors. We write $\Delta_{i, j} \subseteq Y$ for the pull-back of the diagonal $\Delta \subseteq X \times X$ under $\pi_{i, j}$, so that $\Delta_{i, j}$ consists of those points $y=\left(x_{0}, x_{1}, \ldots, x_{p+1}\right) \in Y$ with $x_{i}=x_{j}$.

The basic idea - which goes back to Green [1984b] and has been used repeatedly since (e.g., [Inamdar 1997; Bertram et al. 1991; Lazarsfeld et al. 2011; Hwang and To 2013; Yang 2014]) — is to relate syzygies on $X$ to a suitable union of pairwise diagonals on $Y$. Specifically, let

$$
Z=Z_{p+1}=\Delta_{0,1} \cup \cdots \cup \Delta_{0, p+1} \subseteq X \times X^{p+1}
$$

be the union of the indicated pairwise diagonals, considered as a reduced subscheme. We denote by

$$
q: Z \rightarrow X, \quad \sigma: Z \rightarrow X^{p+1}
$$

the indicated projections.

The importance of this construction for us is given by:

Proposition 1.1. Let $L$ and $B$ be respectively base-point-free and arbitrary line bundles on $X$, and assume (for simplicity) that $H^{1}(X, L)=0$. If the restriction homomorphism

$$
H^{0}\left(Y, B \otimes L^{\otimes p+1}\right) \rightarrow H^{0}\left(Y,\left(B \otimes L^{\bigotimes p+1}\right) \mid Z\right)
$$

is surjective, then

$$
K_{p, 1}(X, B ; L)=0 .
$$

The Proposition was essentially established for instance in [Yang 2014], but it is instructive to give a direct argument. We start with a lemma that will also be useful later:

Lemma 1.2. Writing $I_{Z / Y}$ for the ideal sheaf of $Z$ in $Y$, one has

$$
I_{Z / Y}=I_{\Delta_{0,1} / Y} \cdot I_{\Delta_{0,2} / Y} \cdots I_{\Delta_{0, p+1} / Y}=\bigotimes_{j=1}^{p+1} \pi_{0, j}^{*} I_{\Delta / X \times X} .
$$

Sketch of Proof. This is implicit in [Li 2009, Theorem 1.3], but does not appear there explicitly so we very briefly indicate an argument. The statement is étale local, so we can assume $X=\mathbb{A}^{n}$. By looking at a suitable subtraction map, as in [Lazarsfeld et al. 2011, (1-3)], it then suffices to prove the analogous statement for $Y=X^{p+1}$ with $Z$ being the union of the "coordinate planes"

$$
L_{i}=\left\{\left(x_{1}, \ldots, x_{i}, \ldots, x_{p+1}\right) \mid x_{i}=0 \in \mathbb{A}^{n}\right\}=\left\{\mathbb{A}^{n}\right\} \times \ldots \times\{0\} \times \ldots \times\left\{\mathbb{A}^{n}\right\} \subseteq Y
$$

$(1 \leq i \leq p+1)$. For this one can proceed by induction on $p$, writing out explicitly the equations defining each $L_{i}$. 
Remark 1.3. This is the essential place where we use the hypothesis that $X$ is smooth. We do not know whether the statement of the Lemma remains true for singular $X$.

Proof of Proposition 1.1. To begin with, it is well known (see [Green 1984a]) that $K_{p, 1}(X, B ; L)$ is the cohomology of the Koszul-type complex $\Lambda^{p+1} H^{0}(L) \otimes H^{0}(B) \rightarrow \Lambda^{p} H^{0}(L) \otimes H^{0}(B+L) \rightarrow \Lambda^{p-1} H^{0}(L) \otimes H^{0}(B+2 L)$.

Moreover this cohomology can in turn be interpreted geometrically in terms of the vector bundle $M_{L}$ on $X$ defined (as in the introduction) as the kernel of the evaluation map

$$
\mathrm{ev}_{L}: H^{0}(L) \otimes_{\mathbb{C}} \mathcal{O}_{X} \rightarrow L
$$

Specifically, $M_{L}$ sits in an exact sequence of vector bundles

$$
0 \rightarrow M_{L} \rightarrow H^{0}(L) \otimes \mathcal{O}_{X} \rightarrow L \rightarrow 0
$$

on $X$, and then $K_{p, 1}(X, B ; L)=0$ if and only if the sequence

$$
0 \rightarrow \Lambda^{p+1} M_{L} \otimes B \rightarrow \Lambda^{p+1} H^{0}(L) \otimes B \rightarrow \Lambda^{p} M_{L} \otimes L \otimes B \rightarrow 0
$$

deduced from $(\star)$ is exact on global sections. (See for instance [Green and Lazarsfeld 1986, Lemma 1.10] or [Lazarsfeld 1989].)

On the other hand, consider on $X \times X$ the exact sequence

$$
0 \rightarrow I_{\Delta} \otimes \operatorname{pr}_{2}^{*} L \rightarrow \operatorname{pr}_{2}^{*} L \rightarrow L \otimes \mathcal{O}_{\Delta} \rightarrow 0 .
$$

As in the introduction, this pushes down via $\mathrm{pr}_{1}$ to $(\star)$. Therefore one finds from Lemma 1.2 and the Künneth formula that

$$
q_{*}\left(I_{Z / Y} \otimes B \otimes L^{\otimes p+1}\right)=\left(\bigotimes^{p+1} M_{L}\right) \otimes B,
$$

and moreover the $R^{1} q_{*}$ vanishes thanks to our hypothesis that $H^{1}(L)=0 .{ }^{4}$

Writing

$$
\mathcal{N}=\operatorname{coker}\left(\bigotimes^{p+1} M_{L} \rightarrow \bigotimes^{p+1} H^{0}(L)\right),
$$

${ }^{4}$ The Künneth theorem in play here is the following: let $V_{1} \rightarrow S, \ldots, V_{r} \rightarrow S$ be mappings of schemes over a field, and suppose that $F_{i}$ is a quasicoherent sheaf on $V_{i}$ that is flat over $S$. Write

$$
p_{i}: V_{1} \times{ }_{S} \cdots \times_{S} V_{r} \rightarrow V_{i}, \quad p: V_{1} \times S \cdots \times{ }_{S} V_{r} \rightarrow S
$$

for the natural maps. Then

$$
p_{*}\left(p_{1}^{*} F_{1} \otimes \cdots \otimes p_{r}^{*} F_{r}\right)=p_{1, *} F_{1} \otimes \cdots \otimes p_{r, *} F_{r}
$$

as sheaves on $S$, with analogous Künneth-type computations of the $R^{j} p_{*}$. See for instance [Kempf 1980, Theorem 14] for a simple proof when $S$ is affine, and [EGA III 2 1963, Theorem 6.7.8] for the general case. 
it follows that

$$
q_{*}\left(\left(B \otimes L^{\otimes p+1}\right) \mid Z\right)=\mathcal{N} \otimes B,
$$

and hence the surjectivity of (1-3) is equivalent to asking that

$$
0 \rightarrow\left(\bigotimes^{p+1} M_{L}\right) \otimes B \rightarrow\left(\bigotimes^{p+1} H^{0}(L)\right) \otimes B \rightarrow \mathcal{N} \otimes B \rightarrow 0
$$

be exact on global sections. But since we are in characteristic zero, the exact sequence $(\star \star)$ is a summand of this, and the lemma follows.

Remark 1.4. The argument just completed shows that if in addition $H^{1}(X, B)=0$ then (1-3) is surjective if and only if

$$
H^{1}\left(X,\left(\bigotimes^{p+1} M_{L}\right) \otimes B\right)=0
$$

It remains to relate these considerations to the jet-amplitude of $B$. To this end, keeping notation as in (1-2), set

$$
\mathcal{E}_{B}=\mathcal{E}_{p+1, B}=\sigma_{*}\left(q^{*} B\right) .
$$

This is a torsion-free sheaf of rank $p+1$ on $X^{p+1}$ (since it is the push forward of a line bundle under a finite mapping of degree $p+1$ ), and one has:

Lemma 1.5. (i) Fix a point

$$
\xi=\left(x_{1}, \ldots, x_{p+1}\right) \in X^{p+1},
$$

the $x_{i}$ being (possibly nondistinct) points of $X$, and denote by $\mathcal{E}_{B} \mid \xi$ the fiber of $\mathcal{E}_{B}$ at $\xi$. Then there is a natural identification

$$
\mathcal{E}_{B} \mid \xi=H^{0}\left(X, B \otimes \mathcal{O}_{X} / \mathfrak{m}_{1} \cdots \mathfrak{m}_{p+1}\right),
$$

where $\mathfrak{m}_{i} \subseteq \mathcal{O}_{X}$ is the maximal ideal of $x_{i}$.

(ii) There is a canonical injection

$$
H^{0}(X, B) \hookrightarrow H^{0}\left(X^{p+1}, \mathcal{E}_{B}\right),
$$

giving rise to a homomorphism

$$
\mathrm{ev}_{B}=\operatorname{ev}_{p+1, B}: H^{0}(X, B) \otimes_{\mathbb{C}} \mathcal{O}_{X^{p+1}} \rightarrow \mathcal{E}_{B}
$$

of sheaves on $X^{p+1}$. Under the identification in (i), $\mathrm{ev}_{B}$ is given fiberwise by the natural map

$$
H^{0}(X, B) \rightarrow H^{0}\left(X, B \otimes \mathcal{O}_{X} /\left(\mathfrak{m}_{1} \cdots \mathfrak{m}_{p+1}\right)\right) .
$$


(iii) The homomorphism (1-3) is identified with the map on global sections arising from the sheaf homomorphism

$$
H^{0}(X, B) \otimes_{\mathbb{C}} L^{\unrhd p+1} \rightarrow \mathcal{E}_{B} \otimes L^{\bigotimes p+1} .
$$

on $X^{p+1}$ determined by twisting $\operatorname{ev}_{B}$ by $L^{\bowtie p+1}$.

(iv) The mapping $\operatorname{ev}_{p+1, B}$ is surjective as a homomorphism of sheaves on $X^{p+1}$ if and only if $B$ is p-jet very ample.

Proof. For (i), consider the diagram

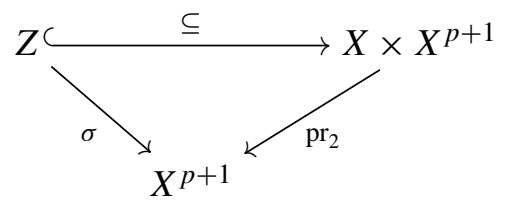

and fix $\xi=\left(x_{1}, \ldots, x_{p+1}\right) \in X^{p+1}$. The scheme-theoretic fiber $\sigma^{-1}(\xi)$ lives naturally as a subscheme of $X$, and Lemma 1.2 implies that it is in fact the scheme defined by the ideal sheaf

$$
\mathfrak{m}_{1} \cdots \mathfrak{m}_{p+1} \subseteq \mathcal{O}_{X}
$$

Therefore, thanks to the projection formula, the fiber $\mathcal{E}_{B} \mid \xi$ of $\mathcal{E}_{B}$ at $\xi$ is identified with

$$
\operatorname{pr}_{2, *}\left(B \otimes \mathcal{O}_{X} /\left(\mathfrak{m}_{1} \cdot \ldots \cdot \mathfrak{m}_{p+1}\right)\right)=H^{0}\left(X, B \otimes \mathcal{O}_{X} /\left(\mathfrak{m}_{1} \cdots \mathfrak{m}_{p+1}\right)\right),
$$

as claimed. For (ii), note that in any event

$$
H^{0}\left(X^{p+1}, \mathcal{E}_{B}\right)=H^{0}\left(X^{p+1}, \sigma_{*} q^{*} B\right)=H^{0}\left(Z, q^{*} B\right) .
$$

On the other hand, each of the irreducible components of $Z$ maps via projection onto $X$, and this gives an inclusion

$$
q^{*}: H^{0}(X, B) \rightarrow H^{0}\left(Z, q^{*} B\right)=H^{0}\left(X \times X^{p+1},\left(\operatorname{pr}_{1}^{*} B\right) \mid Z\right) .
$$

It is evident from the construction that fiber by fiber $\mathrm{ev}_{B}$ is as described, and (iv) is then a consequence of the fact that a morphism of sheaves is surjective if and only it is so on each fiber. Finally, statement (iii) follows from the construction of $\mathcal{E}_{B}$ and $\mathrm{ev}_{B}$.

Remark 1.6. Using the resolution of $\mathcal{O}_{Z}$ appearing in [Yang 2014, p. 4], one can show that in fact

$$
H^{0}\left(X^{p+1}, \mathcal{E}_{B}\right)=H^{0}(X, B) .
$$

However this isn't necessary for the argument. 
Remark 1.7. The reader familiar with [Ein and Lazarsfeld 2015] or Voisin's Hilbert schematic approach to syzygies will recognize that $Z \rightarrow X^{p+1}$ plays the role of the universal family over the Hilbert scheme, and that Proposition 1.1 is the analogue of [Voisin 2002, Lemma 1, p. 369]. The sheaf $\mathcal{E}_{p+1, B}$ plays the role of the vector bundle $E_{p+1, B}$ on the symmetric product appearing in [Ein and Lazarsfeld 2015].

Just as in [Ein and Lazarsfeld 2015], the main result now follows immediately from Serre vanishing.

Proof of Theorem $A$. Assuming that $B$ is $p$-jet very ample, so that $\mathrm{ev}_{p+1, B}$ is surjective, let $\mathcal{M}_{p+1, B}$ denote its kernel:

$$
0 \rightarrow \mathcal{M}_{p+1, B} \rightarrow H^{0}(B) \otimes \mathcal{O}_{X^{p+1}} \rightarrow \mathcal{E}_{B} \rightarrow 0 .
$$

To show that $K_{p, 1}\left(X, B ; L_{d}\right)=0$ it suffices, thanks to Proposition 1.1 and its interpretation in terms of (1-4), to prove that

$$
H^{1}\left(X^{p+1}, \mathcal{M}_{p+1, B} \otimes L_{d}^{\bigotimes p+1}\right)=0
$$

for $d \gg 0$. But this follows immediately from Serre vanishing.

Remark 1.8. It follows from the argument just completed that if $L=L_{d}$ then the surjectivity in Proposition 1.1 holds for $d \gg 0$ if and only if $B$ is $p$-jet very ample. In particular, in view of Remark 1.4 this means that if $H^{1}(X, B)=0$ then the $p$-jet very amplitude of $B$ is equivalent to the vanishing

$$
H^{1}\left(X,\left(\bigotimes^{p+1} M_{L_{d}}\right) \otimes B\right)=0 \quad \text { for } d \gg 0 .
$$

Proof of Corollary $C$. Under the stated hypothesis on $X$, the groups in question are Serre dual to $K_{p, 1}\left(X, B ; L_{d}\right)$ for $d \gg 0$ (see [Green 1984a, §2]). If $B$ fails to be very ample, then a simple argument as in [Eisenbud et al. 2006, Theorem 1.1] shows that $K_{1,1}\left(X, B ; L_{d}\right) \neq 0$ for $d \gg 0$, and therefore the corollary follows from the main theorem.

Remark 1.9. In the case of curves, Rathmann [2016] has given a very interesting argument that leads to an essentially optimal effective version of the asymptotic results of [Ein and Lazarsfeld 2015]: in fact, it suffices that $H^{1}(L)=H^{1}(L-B)=0$. In this spirit, it would be very interesting to find an effective estimate for the positivity of $L$ to guarantee the vanishing of $K_{p, 1}(X, B ; L)$ when $B$ is $p$-jet very ample.

Remark 1.10 (other Koszul cohomology groups). To conclude this section, we briefly summarize what is known about the groups $K_{p, q}\left(X, B ; L_{d}\right)$ for $q \neq 1$. Specifically, fix $B$. Then for $d \gg 0$ :

(i) $K_{p, q}\left(X, B ; L_{d}\right)=0$ for $q \geq n+2$. 
(ii) One has

$$
\begin{aligned}
K_{p, 0}\left(X, B ; L_{d}\right) \neq 0 \Longleftrightarrow 0 \leq p \leq r(B), \text { and } \\
K_{p, n+1}\left(X, B ; L_{d}\right) \neq 0 \Longleftrightarrow r_{d}-n-r\left(K_{X}-B\right) \leq p \leq r_{d}-n .
\end{aligned}
$$

(iii) If $q \geq 2$, then $K_{p, q}\left(X, B ; L_{d}\right)=0$ when $p \leq O(d)$.

(iv) For $1 \leq q \leq n, K_{p, q}\left(X, B ; L_{d}\right) \neq 0$ for

$$
O\left(d^{q-1}\right) \leq p \leq r_{d}-O\left(d^{n-1}\right) .
$$

Statement (i) is a consequence of Castelnuovo-Mumford regularity, while (ii) is due to Green and others. (See [Green 1984a, §3; Ein and Lazarsfeld 2012, Corollary 3.3, §5].) Assertion (ii) follows for instance from [Ein and Lazarsfeld 1993], while (iv) is the main result of [Ein and Lazarsfeld 2012]. Furthermore, it is conjectured in [Ein and Lazarsfeld 2012] that if $q \geq 2$, then $K_{p, q}\left(X, B ; L_{d}\right)=0$ for $p \leq O\left(d^{q-1}\right)$.

\section{A nonvanishing theorem}

This section is devoted to the proof of Theorem B from the introduction. Recall the statement:

Theorem 2.1. Assume that the nonsingular projective variety $X$ carries an effective $(p+1)$-cycle $w=x_{1}+\cdots+x_{p+1}$ consisting of $p+1$ distinct points $x_{1}, \ldots, x_{p+1} \in X$ that fail to impose independent conditions on $H^{0}(X, B)$. Then

$$
K_{p, 1}\left(X, B ; L_{d}\right) \neq 0 \quad \text { for } d \gg 0 .
$$

The argument is somewhat technical, so before launching into it we would like to outline the rough strategy. As in the case $p=0$ discussed in the introduction, in principle we would like to find a map of sheaves on $\operatorname{Sym}^{p+1}(X)$ depending on $B$ say $a_{B}: \mathcal{A}_{1} \rightarrow \mathcal{A}_{2}$ - having the property that

$$
K_{p, 1}\left(X, B ; L_{d}\right)=\operatorname{coker}\left(H^{0}\left(\mathcal{A}_{1} \otimes \mathcal{N}\left(L_{d}\right)\right) \rightarrow H^{0}\left(\mathcal{A}_{2} \otimes \mathcal{N}\left(L_{d}\right)\right)\right),
$$

where $\mathcal{N}\left(L_{d}\right)$ is a line bundle whose positivity grows suitably with $d$. Ideally - as in equation $(* * *)$ from the introduction - we would be able to see that $a_{B}$ cannot be surjective as a map of sheaves if $B$ is not $p$-jet very ample, and then one could hope to apply Serre vanishing to conclude that $K_{p, 1}\left(X, B ; L_{d}\right)$ cannot vanish for $d \gg 0$. Unfortunately we do now know whether such a construction is possible. Instead, what we do in effect is to use the ideas of Yang [2014] to construct a map $\alpha_{B}$, and show that the nonvanishing of $K_{p, 1}$ is implied by the nonvanishing of a certain quotient sheaf of $\mathcal{A}_{2}$. We show that a reduced $(p+1)$-cycle that fails to impose independent conditions on $H^{0}(X, B)$ must appear in the support of this quotient, and this leads to the stated nonvanishing. 
We start by recalling the results of Yang [2014] interpreting $K_{p, 1}$ as an equivariant cohomology group. Consider then a very ample line bundle $L$ on the smooth complex projective variety $X$. Then the symmetric group $S_{p+1}$ acts in two ways on the bundle $L^{\bigotimes p+1}$ on $X^{p+1}$, namely via the symmetric and the alternating characters. Denote these $S_{p+1}$-bundles on $X^{p+1}$ by

$$
L^{\bigotimes p+1, \text { sym }} \quad \text { and } \quad L^{\bigotimes p+1, \text { alt }},
$$

respectively. Now let $S_{p+1}$ act on $X \times X^{p+1}$ via the trivial action on the first factor, so that the union of pairwise diagonals $Z \subseteq X \times X^{p+1}$ defined in (1-1) becomes an $S_{p+1}$-subspace. It is established in [Yang 2014, Theorem 3] that if

$$
H^{i}(X, m L)=H^{i}(X, B+m L)=0 \text { for } i, m>0,
$$

then $K_{p, 1}(X, B ; L)$ is identified with the cokernel of the restriction mapping

$$
H_{S_{p+1}}^{0}\left(X \times X^{p+1}, \operatorname{pr}_{1}^{*} B \otimes \operatorname{pr}_{2}^{*} L^{\bigotimes p+1, \text { alt }}\right) \rightarrow H_{S_{p+1}}^{0}\left(Z, \operatorname{pr}_{1}^{*} B \otimes \operatorname{pr}_{2}^{*} L^{\bigotimes p+1, \text { alt }} \otimes \mathcal{O}_{Z}\right)
$$

on $S_{p+1}$-equivariant cohomology groups..$^{5}$ One can think of this as a precision and strengthening of Proposition 1.1. Following the line of attack of Section 1, the plan is to study these groups by modding out by the symmetric group and pushing down to the symmetric product.

To this end, denote by $\operatorname{Sym}^{p+1}(X)$ the $(p+1)$-st symmetric product of $X$, which we view as parametrizing zero-cycles of degree $p+1$, and write

$$
\pi: X^{p+1} \rightarrow \operatorname{Sym}^{p+1}(X)
$$

${ }^{5}$ For the theory of equivariant cohomology groups and pushforwards, see [Grothendieck 1957, Chapter 5]. What we need can be summarized as follows. Let $G$ be a finite group acting on a complex projective variety $V$, and suppose a coherent sheaf $F$ on $X$ together with an action of $G$ on $F$ are given. Then one can define equivariant cohomology groups $H_{G}^{j}(V, F)$. While this isn't how they are initially constructed, one can show that

$$
H_{G}^{j}(V, F)=H^{j}(V, F)^{G},
$$

the group on the right being the $G$-invariant subspace of $H^{j}(V, F)$ under the natural action of $G$ on this cohomology group [Grothendieck 1957, p. 202], and for practical purposes one can take this as the definition. Writing

$$
\pi: V \rightarrow V / G \stackrel{\text { def }}{=} W,
$$

one also has an action of $G$ on $\pi_{*} F$. The $G$-equivariant direct image of $F$ can be interpreted as

$$
\pi_{*}^{G} F=\left(\pi_{*}(F)\right)^{G},
$$

and one can show that

$$
H_{G}^{j}(V, F)=H^{j}\left(W, \pi_{*}^{G} F\right)
$$


for the quotient map. The equivariant pushforward of the line bundles in (2-2) determine respectively a line bundle and torsion-free sheaf of rank one

$$
\mathcal{S}_{p+1}(L) \stackrel{\text { def }}{=} \pi_{*}^{S_{p+1}}\left(L^{\square p+1, \text { sym }}\right), \quad \mathcal{N}_{p+1}(L) \stackrel{\text { def }}{=} \pi_{*}^{S_{p+1}}\left(L^{\otimes p+1, \text { alt }}\right)
$$

on $\operatorname{Sym}^{p+1}(X)$. One has

$$
\begin{aligned}
& H^{0}\left(\operatorname{Sym}^{p+1}(X), \mathcal{S}_{p+1}(L)\right)=\operatorname{Sym}^{p+1} H^{0}(X, L), \\
& H^{0}\left(\operatorname{Sym}^{p+1}(X), \mathcal{N}_{p+1}(L)\right)=\Lambda^{p+1} H^{0}(X, L),
\end{aligned}
$$

and for any line bundle $A$ on $X$ :

$$
\mathcal{S}(L \otimes A)=\mathcal{S}(L) \otimes \mathcal{S}(A), \quad \mathcal{N}(L \otimes A)=\mathcal{N}(L) \otimes \mathcal{S}(A) .
$$

Moreover $\mathcal{S}(A)$ is ample if $A$ is.

Proof of Theorem 2.1. Note to begin with that the symmetric group $S_{p+1}$ acts on each of the spaces appearing in diagram (1-5). Taking the quotients yields the diagram

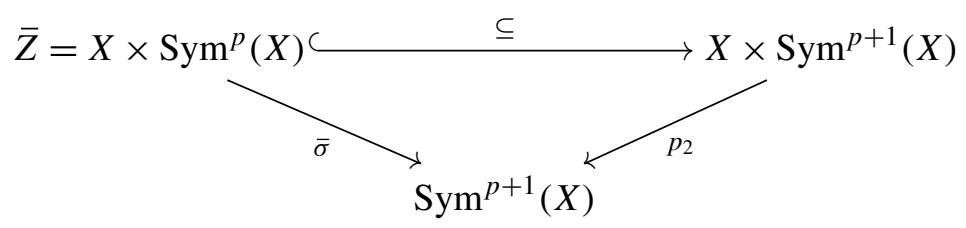

where $\bar{\sigma}$ is the addition map,

$$
p_{1}: X \times \operatorname{Sym}^{p+1}(X) \rightarrow X, \quad p_{2}: X \times \operatorname{Sym}^{p+1}(X) \rightarrow \operatorname{Sym}^{p+1}(X)
$$

are the projections, and the inclusion on the top line is given by $(x, w) \mapsto(x, x+w)$. One has

$$
(1 \times \pi)_{*}^{S_{p+1}}\left(\operatorname{pr}_{1}^{*} B \otimes \mathrm{pr}_{2}^{*} L^{\otimes p+1, \text { alt }}\right)=p_{1}^{*} B \otimes p_{2}^{*} \mathcal{N}(L) .
$$

Now define

$$
\mathcal{G}(B ; L)=(1 \times \pi)_{*}^{S_{p+1}}\left(\operatorname{pr}_{1}^{*} B \otimes \operatorname{pr}_{2}^{*} L^{\otimes p+1, \text { alt }} \otimes \mathcal{O}_{Z}\right) .
$$

Pushing forward the restriction to $Z$ gives rise to a natural surjective mapping

$$
\varepsilon(B ; L): p_{1}^{*} B \otimes p_{2}^{*} \mathcal{N}(L) \rightarrow \mathcal{G}(B ; L)
$$

of sheaves on $X \times \operatorname{Sym}^{p+1}(X)$. Thanks to [Grothendieck 1957, §5.2], the groups appearing in (2-4) are given by the global sections of the sheaves in (2-7), and hence under the vanishing hypothesis $(2-3), K_{p, 1}(X, B ; L)$ is computed as the cokernel

$$
K_{p, 1}(X, B ; L)=\operatorname{coker}\left(H^{0}(\varepsilon(B ; L))\right)
$$


on global sections determined by $\varepsilon(B ; L)$. Hence we are reduced to showing that under the hypothesis of the theorem, $\varepsilon\left(B ; L_{d}\right)$ cannot be surjective on global sections when $d \gg 0$.

The next step is to form and study the push-forward of (2-7) to $\operatorname{Sym}^{p+1}(X)$. To begin with, define $\mathcal{F}(B ; L)=p_{2, *} \mathcal{G}(B ; L)$ and $\delta(B ; L)=p_{2, *} \varepsilon(B ; L)$. This gives rise to a morphism

$$
\delta(B ; L): H^{0}(X, B) \otimes_{\mathbb{C}} \mathcal{N}(L) \rightarrow \mathcal{F}(B ; L)
$$

of sheaves on $\operatorname{Sym}^{p+1}(X)$ with the property that

$$
K_{p, 1}(X, B ; L)=\operatorname{coker}\left(H^{0}(\delta(B, L))\right) .
$$

We wish to study the geometry of this mapping assuming that $B$ does not impose independent conditions on all reduced cycles. We assert that there is a natural homomorphism

$$
t:\left(p_{1}^{*} B \otimes p_{2}^{*} \mathcal{N}(L) \otimes \mathcal{O}_{\bar{Z}}\right) \rightarrow \mathcal{G}(B ; L)
$$

which is an isomorphism on the smooth locus of $\bar{Z}$. Grant this for the time being. By the projection formula one has

$$
p_{2, *}\left(p_{1}^{*} B \otimes p_{2}^{*} \mathcal{N}(L) \otimes \mathcal{O}_{\bar{Z}}\right)=\bar{\sigma}_{*}\left(p_{1}^{*} B\right) \otimes \mathcal{N}(L),
$$

and then taking direct images in (2-7) and (2-8), one arrives at a diagram

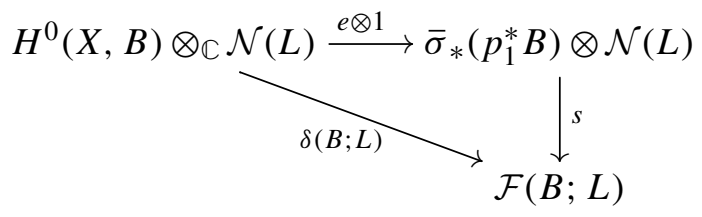

where $s$ is an isomorphism over the smooth locus of $\operatorname{Sym}^{p+1}(X)$.

Now fix a reduced zero-cycle $w \in \operatorname{Sym}^{p+1}(X)$ that fails to impose independent conditions on $H^{0}(X, B)$, and let $\xi \subseteq X$ be the corresponding subscheme of length $p+1$. We can identify the fiber of the morphism

$$
e: H^{0}(X, B) \otimes \mathcal{O}_{\operatorname{Sym}^{p+1}(X)} \rightarrow \bar{\sigma}\left(p_{1}^{*} B\right)
$$

appearing in (2-9) at $w$ with the evaluation $H^{0}(X, B) \rightarrow H^{0}\left(X, B \otimes \mathcal{O}_{\xi}\right)$. Writing $\mathcal{K}=\operatorname{coker}(e)$, so that

$$
\operatorname{coker}(e \otimes 1)=\mathcal{K} \otimes \mathcal{N}(L),
$$

it follows that $w \in \operatorname{supp}(\mathcal{K} \otimes \mathcal{N}(L))$. But thanks to (2-5) and Serre vanishing, $\bar{\sigma}_{*}\left(p_{1}^{*} B\right) \otimes \mathcal{N}\left(L_{d}\right)$ is globally generated and

$$
H^{0}\left(\bar{\sigma}\left(p_{1}^{*} B\right) \otimes \mathcal{N}\left(L_{d}\right)\right) \rightarrow H^{0}\left(\mathcal{K} \otimes \mathcal{N}\left(L_{d}\right)\right)
$$


is surjective when $d \gg 0$. On the other hand, $s$ is an isomorphism in a neighborhood of $w$ since $w$ is reduced, and it then follows that the map

$$
H^{0}\left(\mathcal{K} \otimes \mathcal{N}\left(L_{d}\right)\right) \rightarrow H^{0}\left(\operatorname{coker}\left(\delta\left(B ; L_{d}\right)\right)\right)
$$

is nonzero when $d \gg 0$. In other words, we have a commutative diagram

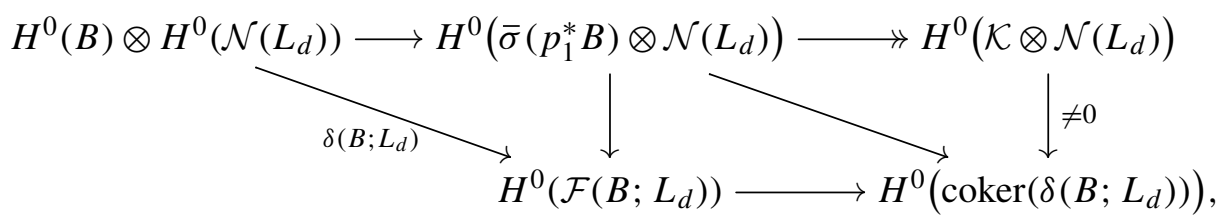

with exact top row, in which the right-hand diagonal mapping, and hence also the bottom homomorphism, are nonzero. Therefore $\delta\left(B ; L_{d}\right)$ cannot be surjective on global sections when $d \gg 0$, as required.

It remains to construct the homomorphism $t$ appearing in (2-8). To this end, let

$$
\widehat{Z}=\bar{Z} \times{ }_{X \times \operatorname{Sym}^{p+1}(X)}\left(X \times X^{p+1}\right) .
$$

The projection formula gives an isomorphism $(1 \times \pi)_{*}\left(\left(\mathrm{pr}_{1}^{*} B \otimes \mathrm{pr}_{2}^{*} L^{\bigotimes p+1, \text { alt }}\right) \otimes \mathcal{O}_{\widehat{Z}}\right)=\left((1 \times \pi)_{*}\left(\operatorname{pr}_{1}^{*} B \otimes \mathrm{pr}_{2}^{*} L^{\bigotimes p+1, \text { alt }}\right)\right) \otimes \mathcal{O}_{\bar{Z}}$, which, upon taking $S_{p+1}$ invariants, yields

$$
(1 \times \pi)_{*}^{S_{p+1}}\left(\operatorname{pr}_{1}^{*} B \otimes \operatorname{pr}_{2}^{*} L^{\boxplus p+1, \text { alt }} \otimes \mathcal{O}_{\widehat{Z}}\right)=\left(p_{1}^{*} B \otimes p_{2}^{*} \mathcal{N}(L)\right) \otimes \mathcal{O}_{\bar{Z}} .
$$

On the other hand, as a set $\widehat{Z}$ consists of those points

$$
\left(x_{0},\left(x_{1}, \ldots, x_{p+1}\right)\right) \in X \times X^{p+1}
$$

having the property that $x_{0}$ appears in the cycle $x_{1}+\cdots+x_{p+1}$. In other words, $\widehat{Z}$ and $Z$ coincide set-theoretically. Since $Z$ is reduced this implies that $Z=\widehat{Z}_{\text {red }}$, and in particular $Z$ is a subscheme of $\widehat{Z}$. Thus there is a natural surjective map

$$
\left(\operatorname{pr}_{1}^{*} B \otimes \operatorname{pr}_{2}^{*} L^{\unrhd p+1, \text { alt }}\right) \otimes \mathcal{O}_{\widehat{Z}} \rightarrow\left(\operatorname{pr}_{1}^{*} B \otimes \operatorname{pr}_{2}^{*} L^{\unrhd p+1, \text { alt }}\right) \otimes \mathcal{O}_{Z}
$$

which is an isomorphism over the smooth locus of $Z$, and taking direct images gives (2-8).

Remark 2.2. It would be very interesting to give a necessary and sufficient condition for the nonvanishing of $K_{p, 1}\left(X, B ; L_{d}\right)$ for $d \gg 0$. Keeping the notation of the previous proof, the issue is to determine when $\delta(B ; L)$ has a nonzero cokernel. It is conceivable that the failure of $B$ to be $p$-jet very ample suffices, but the question seems somewhat difficult to analyze. Already the case $\operatorname{dim} X=2$ would be interesting. 
Remark 2.3. Recall that a line bundle $B$ on a smooth variety $X$ is said to be $p$-very ample if every finite subscheme of length $p+1$ imposes independent conditions on $H^{0}(X, B)$. When $\operatorname{dim} X=1$ this is the same as jet-amplitude, but when $\operatorname{dim} X \geq 2$ it is a strictly weaker condition in general. A quick way to see this is to recall that if $A$ is an ample line bundle on a smooth surface $X$, then $B_{p}=K_{X}+(p+3) A$ is always $p$-very ample thanks to a theorem of Beltrametti, Francia and Sommese [1989]. On the other hand, the $p$-jet amplitude of $B_{p}$ for $p \gg 0$ would imply that the Seshadri constant $\varepsilon(A ; x)$ is very close to 1 for every point $x \in X$ (see [Lazarsfeld 1997, Proposition 5.10]). Hence any line bundle $A$ for which there exist points with small Seshadri constant gives rise to examples of the required sort.

\section{Acknowledgements}

We are grateful to Claudiu Raicu and Bernd Sturmfels for valuable discussions. We particularly profited from conversations with B. Purnaprajna, who suggested to us that one could work on a Cartesian rather than a symmetric product to establish the vanishing we wanted.

\section{References}

[Beltrametti et al. 1989] M. Beltrametti, P. Francia, and A. J. Sommese, "On Reider's method and higher order embeddings”, Duke Math. J. 58:2 (1989), 425-439. MR 1016428 Zbl 0702.14010

[Bertram et al. 1991] A. Bertram, L. Ein, and R. Lazarsfeld, "Vanishing theorems, a theorem of Severi, and the equations defining projective varieties", J. Amer. Math. Soc. 4:3 (1991), 587-602. MR 1092845 Zbl 0762.14012

[EGA III 2 1963] A. Grothendieck, "Eléments de géométrie algébrique, III: Étude cohomologique des faisceaux cohérents, II”, Inst. Hautes Études Sci. Publ. Math. 17 (1963), 5-91. MR 0163911 Zbl 0122.16102

[Ein and Lazarsfeld 1993] L. Ein and R. Lazarsfeld, "Syzygies and Koszul cohomology of smooth projective varieties of arbitrary dimension", Invent. Math. 111:1 (1993), 51-67. MR 1193597 Zbl 0814.14040

[Ein and Lazarsfeld 2012] L. Ein and R. Lazarsfeld, "Asymptotic syzygies of algebraic varieties", Invent. Math. 190:3 (2012), 603-646. MR 2995182 Zbl 1262.13018

[Ein and Lazarsfeld 2015] L. Ein and R. Lazarsfeld, "The gonality conjecture on syzygies of algebraic curves of large degree", Publ. Math. Inst. Hautes Études Sci. 122 (2015), 301-313. MR 3415069 Zbl 1342.14070

[Eisenbud et al. 2006] D. Eisenbud, C. Huneke, and B. Ulrich, "The regularity of Tor and graded Betti numbers", Amer. J. Math. 128:3 (2006), 573-605. MR 2230917 Zbl 1105.13017

[Green 1984a] M. L. Green, "Koszul cohomology and the geometry of projective varieties", $J$. Differential Geom. 19:1 (1984), 125-171. MR 739785 Zbl 0559.14008

[Green 1984b] M. L. Green, "Koszul cohomology and the geometry of projective varieties, II", J. Differential Geom. 20:1 (1984), 279-289. MR 772134 Zbl 0559.14009

[Green and Lazarsfeld 1986] M. Green and R. Lazarsfeld, "On the projective normality of complete linear series on an algebraic curve”, Invent. Math. 83:1 (1986), 73-90. MR 813583 Zbl 0594.14010 
[Grothendieck 1957] A. Grothendieck, "Sur quelques points d'algèbre homologique", Tôhoku Math. J. (2) 9 (1957), 119-221. MR 0102537 Zbl 0118.26104

[Hwang and To 2013] J.-M. Hwang and W.-K. To, "Syzygies of compact complex hyperbolic manifolds”, J. Algebraic Geom. 22:1 (2013), 175-200. MR 2993051 Zbl 1262.14008

[Inamdar 1997] S. P. Inamdar, “On syzygies of projective varieties”, Pacific J. Math. 177:1 (1997), 71-76. MR 1444773 Zbl 0898.14015

[Kempf 1980] G. R. Kempf, "Some elementary proofs of basic theorems in the cohomology of quasicoherent sheaves”, Rocky Mountain J. Math. 10:3 (1980), 637-645. MR 590225

[Lazarsfeld 1989] R. Lazarsfeld, "A sampling of vector bundle techniques in the study of linear series", pp. 500-559 in Lectures on Riemann surfaces (Trieste, 1987), edited by M. Cornalba et al., World Sci., Teaneck, NJ, 1989. MR 1082360 Zbl 0800.14003

[Lazarsfeld 1997] R. Lazarsfeld, "Lectures on linear series", pp. 161-219 in Complex algebraic geometry (Park City, UT, 1993), edited by J. Kollár, IAS/Park City Math. Ser. 3, Amer. Math. Soc., Providence, RI, 1997. MR 1442523

[Lazarsfeld et al. 2011] R. Lazarsfeld, G. Pareschi, and M. Popa, "Local positivity, multiplier ideals, and syzygies of abelian varieties", Algebra Number Theory 5:2 (2011), 185-196. MR 2833789 Zbl 1239.14035

[Li 2009] L. Li, "Wonderful compactification of an arrangement of subvarieties", Michigan Math. J. 58:2 (2009), 535-563. MR 2595553 Zbl 1187.14060

[Rathmann 2016] J. Rathmann, “An effective bound for the gonality conjecture”, preprint, 2016. arXiv 1604.06072

[Voisin 2002] C. Voisin, "Green's generic syzygy conjecture for curves of even genus lying on a $K 3$ surface”, J. Eur. Math. Soc. (JEMS) 4:4 (2002), 363-404. MR 1941089 Zbl 1080.14525

[Voisin 2005] C. Voisin, “Green's canonical syzygy conjecture for generic curves of odd genus", Compos. Math. 141:5 (2005), 1163-1190. MR 2157134 Zbl 1083.14038

[Yang 2014] D. H. Yang, " $S_{n}$-equivariant sheaves and Koszul cohomology”, Res. Math. Sci. 1 (2014), Art. 10, 6. MR 3375645

Communicated by David Eisenbud

Received 2015-12-23 Revised 2016-06-29 Accepted 2016-08-21

ein@uic.edu Department of Mathematics, University of Illinois, Chicago, Chicago, IL 60607, United States

robert.lazarsfeld@stonybrook.edu

Department of Mathematics, Stony Brook University, Stony Brook, NY 11794, United States

dhy@mit.edu

Department of Mathematics, MIT, Building 2,

182 Memorial Dr., Cambridge, MA 02139, United States 


\section{Algebra \& Number Theory}

msp.org/ant

\section{EDITORS}

MANAGING EDITOR

Bjorn Poonen

Massachusetts Institute of Technology

Cambridge, USA

\author{
EDITORIAL BOARD CHAIR \\ David Eisenbud \\ University of California \\ Berkeley, USA
}

BOARD OF EDITORS

$\begin{aligned} \text { Dave Benson } & \text { University of Aberdeen, Scotland } & \text { Susan Montgomery } & \text { University of Southern California, USA } \\ \text { Richard E. Borcherds } & \text { University of California, Berkeley, USA } & \text { Shigefumi Mori } & \text { RIMS, Kyoto University, Japan } \\ \text { John H. Coates } & \text { University of Cambridge, UK } & \text { Raman Parimala } & \text { Emory University, USA } \\ \text { J-L. Colliot-Thélène } & \text { CNRS, Université Paris-Sud, France } & \text { Jonathan Pila } & \text { University of Oxford, UK } \\ \text { Brian D. Conrad } & \text { Stanford University, USA } & \text { Anand Pillay } & \text { University of Notre Dame, USA } \\ \text { Hélène Esnault } & \text { Freie Universität Berlin, Germany } & \text { Victor Reiner } & \text { University of Minnesota, USA } \\ \text { Hubert Flenner } & \text { Ruhr-Universität, Germany } & \text { Peter Sarnak } & \text { Princeton University, USA } \\ \text { Sergey Fomin } & \text { University of Michigan, USA } & \text { Joseph H. Silverman } & \text { Brown University, USA } \\ \text { Edward Frenkel } & \text { University of California, Berkeley, USA } & \text { Michael Singer } & \text { North Carolina State University, USA } \\ \text { Andrew Granville } & \text { Université de Montréal, Canada } & \text { Vasudevan Srinivas } & \text { Tata Inst. of Fund. Research, India } \\ \text { Joseph Gubeladze } & \text { San Francisco State University, USA } & \text { J. Toby Stafford } & \text { University of Michigan, USA } \\ \text { Roger Heath-Brown } & \text { Oxford University, UK } & \text { Ravi Vakil } & \text { Stanford University, USA } \\ \text { Craig Huneke } & \text { University of Virginia, USA } & \text { Michel van den Bergh } & \text { Hasselt University, Belgium } \\ \text { Kiran S. Kedlaya } & \text { Univ. of California, San Diego, USA } & \text { Marie-France Vignéras } & \text { Université Paris VII, France } \\ \text { János Kollár } & \text { Princeton University, USA } & \text { Kei-Ichi Watanabe } & \text { Nihon University, Japan } \\ \text { Yuri Manin } & \text { Northwestern University, USA } & \text { Efim Zelmanov } & \text { University of California, San Diego, USA } \\ \text { Philippe Michel } & \text { École Polytechnique Fédérale de Lausanne } & \text { Shou-Wu Zhang } & \text { Princeton University, USA }\end{aligned}$

PRODUCTION

production@msp.org

Silvio Levy, Scientific Editor

See inside back cover or msp.org/ant for submission instructions.

The subscription price for 2016 is US $\$ 290$ /year for the electronic version, and $\$ 485 /$ year (+\$55, if shipping outside the US) for print and electronic. Subscriptions, requests for back issues and changes of subscribers address should be sent to MSP.

Algebra \& Number Theory (ISSN 1944-7833 electronic, 1937-0652 printed) at Mathematical Sciences Publishers, 798 Evans Hall \#3840, c/o University of California, Berkeley, CA 94720-3840 is published continuously online. Periodical rate postage paid at Berkeley, CA 94704, and additional mailing offices.

ANT peer review and production are managed by EditFLow ${ }^{\circledR}$ from MSP.

\section{PUBLISHED BY}

- mathematical sciences publishers

nonprofit scientific publishing

http://msp.org/

() 2016 Mathematical Sciences Publishers 


\section{Algebra \& Number Theory}

Volume $10 \quad$ No. $9 \quad 2016$

Torsion pour les variétés abéliennes de type I et II

MARC HINDRY and NICOLAS RATAZZI

Galois-generic points on Shimura varieties

ANNA CADORET and ARNO KRET

Extremality of loci of hyperelliptic curves with marked Weierstrass points

DAWEI CHEN and NiCOLA TARASCA

$\bar{\Re}_{15}$ is of general type

GREGOR BRUNS

A vanishing theorem for weight-one syzygies

LAWRENCE EIN, ROBERT LAZARSFELD and DAVID YANG

1983

Effective cones of cycles on blowups of projective space

IZZET COSKUN, JOHN LESIEUTRE and JOHN CHRISTIAN OTTEM

Cluster algebras and category $\mathrm{O}$ for representations of Borel subalgebras of quantum affine algebras

DAVID HERNANDEZ and BERNARD LECLERC 